\title{
A REVIEW ON MUCOADHESIVE VAGINAL DRUG DELIVERY SYSTEM
}

\author{
Ms.S.A. Chore* and S.J. Dighade \\ Institute of Pharmacy and Research, \\ Badnera-Amaravati, Maharashtra, India.
}

\begin{abstract}
Vaginitis is a very common gynaecological problem in women of all age groups. There are three types of infectious vaginitis: candidiasis, trichomoniasis, and bacterial vaginosis. Vaginitis can be caused by single or mixed vaginal infections. Approximately $30 \%$ of women suffering from vaginitis problem. Some drugs are poorly absorbed after the oral administration. The vaginal route appears to be highly appropriate for mucoadhesive drug delivery systems in order to retain drugs for treating largely local conditions, or for use in contraception. In particular, protection against sexually-transmitted diseases. To prolong the residence time in the vaginal cavity, mucoadhesive therapeutic systems have been developed in the form of semi-solid and solid dosage forms. Over the past few decades, mucosal drug delivery has received a great deal of attention. Mucoadhesive dosage forms may be designed to enable prolonged retention at the site of application, providing a controlled rate of drug release for improved therapeutic outcome. Application of dosage forms to mucosal surfaces may be of benefit to drug molecules not amenable to the oral route, such as those that undergo acid degradation or extensive first-pass metabolism. The mucoadhesive ability of a dosage form is dependent upon a variety of factors, including the nature of the mucosal tissue and the physicochemical properties of the polymeric formulation. This review article aims to provide an overview of the various aspects of vaginal drug delivery, factors affecting vaginal drug delivery, vaginal absorption of drugs, various mucoadhesive drug delivery systems (buccal, nasal, ocular, gastro, vaginal, and rectal), Various dosage form of mucoadhesive drug delivery system.
\end{abstract}

Keywords: Intra vaginal delivery, vaginal formulation, vaginal tablet and vaginal ring.

\section{INTRODUCTION}

The vaginal route is commonly used for the administration of locally acting drugs such as antimicrobials, labor-inducing agents, spermicidal agents, prostaglandins and steroids. Moreover, the administration of drugs for systemic effects via the vagina is also feasible In comparison to all other mucosal membranes, the vaginal mucosa offers the advantage that drug delivery systems can remain for the longest time period at the site of application. Moreover; vaginal rings can remain on the vaginal mucosa even for months, which renders this route of noninvasive administration unique and provides promising opportunity for more efficient and convenient therapies. The full potential of these drug delivery systems which provide a prolonged residence time, however, has yet to be realized. An overview of state-of-the-art vaginal retentive delivery systems shall be given within this review. It shall provide the basis for the development of novel more effective vaginal formulations guaranteeing prolonged residence times. The vaginal ecosystem is a finely balanced environment maintained by a complex interaction among vaginal flora, microbial by-products, estrogens and host factors. The vagina is usually resistant to infection for two reasons- marked 
acidity and a thick protective epithelium. Insults that affect the vaginal microbiology, vaginal epithelium, or vaginal $\mathrm{pH}$ lead to an increased susceptibility to vaginal infections. The administration of a dosage form via vaginal route can result in either local or systemic delivery or both. The successful delivery of the drugs through vagina represents a pharmaceutical challenge for several reasons. The major reason being, vagina has unique features in terms of microflora, $\mathrm{pH}$, hormonal cyclic changes, permeability perfusion time etc. These factors must be considered during the development and evaluation of vaginal drug delivery systems. A well designed vaginal product depends on the vehicles (base) that effectively deliver the active pharmaceutical ingredient (drug) in a pattern that does not destroy the pharmacological agent and is simultaneously safe for the patient. There are varieties of products available in the market for vaginal administration that includes creams, tablets, gels, pessaries, suppositories, films, rings and douches. The choice of route of administration is primarily influenced by location of the biological target. If the target is external or easily accessed then local delivery of the medication is effective approach. Literature survey has revealed that locally acting drugs used in vagina includes antibacteria, antiprotozoal, antifungal and antiviral. Whereas, for systemic effects the drugs used includes bromocriptine, sildenafil, oxytocin, calcitonin, LHRH, insulin, human growth hormone.

\section{Advantage of vaginal drug delivery}

1. The vagina may serve as a better route for the delivery of drugs due to the paucity of drug metabolism and the avoidance of the liver first-past effect.

2. A reduction in hepatic side effects of steroids used in hormone replacement therapy or contraception period.

3. Avoidance of the inconvenience caused by pain, tissue damage and risk of infections which are associated with parenteral routes.

4. Ease of self-insertion and removal of the dosage form is possible.

\section{Limitations of vaginal drug delivery systems}

Apart from above mentioned advantages this route has several disadvantages too which is listed as follows

- This drug delivery system is gender specific.
- The vaginal route is less preferred because of inconvenience.

- The permeability of the vagina is strongly influenced by the estrogens concentration, which can influence the pharmacokinetics of drugs.

- The amount of vaginal fluid of an adult woman was reported to be in the range of 2-3 g (gram)/24 $\mathrm{h}$ (hour) and this amount is decreasing with increasing age which can affect the vaginal absorption of drugs.

- The $\mathrm{pH}$ of the vaginal fluid is also a factor which affects the drug absorption as the unionized drugs absorbed.

Vaginal, Anatomy Histology and
Physiology
The vagina plays a major role in reproductionand it is an important organ of the reproductive tract. It is a strong canal of muscle and approx. $7.5 \mathrm{~cm}$ long that extends from the uterus to the vestibule of the external genitalia (fig.1). For enough elasticity the vaginal wall is crosswise fold (Fig. 1). The vagina is positioned between rectum, bladder and urethra. The function and construction is significantly different to the intestinal wall. In contrast to the intestine there is no peristaltic motion but it is also not rigid. The vaginal wall consists of three layers: the epithelial layer, the muscular coat and tunica adventitia. A cell turnover of about 10-15 layers is estimated to be in the order of 7 days. The epithelium is a noncornified, stratified squamous epithelium. The thickness is dependent on age. With hormonal activity the vaginal epithelium increases in thickness and is highest in the proliferative stage and reaches the highest glycogen content during ovulation. Although the cyclic changes of the vaginal epithelium are less pronounced than of the endometrium, although differential cytology of the vaginal epithelium can be used to identify the cycle stages. As can be seen the epithelium thickness is also dependent on the different life stages like newborn, child, adult and menopause (Fig. 1C. The main blood supply to the vagina is through the vaginal branch of the uterine artery. The vagina has unique features in terms of microflora, $\mathrm{pH}$ and cyclic changes, and these factors must be considered during the development and evaluation of vaginal delivery systems (Fig. 1: Schematic drawing of the vagina).

Factors affecting vaginal Drug Delivery
system 
The ecology of the vagina is influenced by factors such as the glycogen content of epithelial cells, glucose, $\mathrm{pH}$, hormonal levels, trauma during sexual intercourse, birth-control method, age, antimicrobial treatment and delivery. The vaginal flora is a dynamic system mainly consisting of Lactobacillus which is the most prevalent organism in the vaginal environment together with many other facultative and obligate aerobes and anaerobes. Vaginal biopsies were found to be highly suitable for demonstrating distribution of vaginal mucosa. The glycogen content of superficial vaginal epithelium showed a tendency to increase throughout the cycle and then fall in the late premenstrual phase. This was according the estrogen excretion through the cycle. As the estrogen production decreases during the pre-menopause and on going menopause there is a permanent decrease in the vaginal glycogen content.

\section{Vaginal pH}

Normal microflora predominantly lactobacilli produce sufficient lactic acid to acidify vaginal secretions to $\mathrm{pH}$ 3.5-4.5. This value is maintained by the lactobacilli which convert glycogen from exfoliated epithelial cells into lactic acid. The $\mathrm{pH}$ changes with age, stages of menstrual cycle, infections and sexual arousal. In most of the women a $\mathrm{pH}$ gradient exists in the vagina. Menstrual, cervical and uterine secretions and semen act as alkalising agents and increase the $\mathrm{pH}$. The $\mathrm{pH}$ plays also a role in amount of drug absorption and is important for drug delivery systems. The conception of such a drug delivery system should correlate to the amount of the vaginal fluid in order to exclude a loosening of the epithelium barrier.

\section{Cyclic changes}

As mentioned before the changes in hormone levels (especially estrogen) during the menstrual cycle lead to alterations in the thickness of the epithelial cell layer, width of intercellular channels, $\mathrm{pH}$ and secretions. The variations in enzyme activity (endopeptidases and aminopeptidases) with hormonal changes further complicate the problem of achieving consistent drug delivery.

\section{Enzyme Activity}

The specific enzymatic activity of four different amino peptidases in vaginal homogenates decreases in the order: sheep $>$ guinea pig > rabbit $\geq$ human $\geq$ rat. The human genital tract has lower enzymatic activity leading to less degradation of protein and peptide drugs in the vagina than the gastrointestinal tract.

\section{Micro flora}

The factors which influence the ecology of the vagina are glycogen content of epithelial cells, glucose, $\mathrm{pH}$, hormonal levels and trauma during sexual intercourse, birth-control method, age, antimicrobial treatment and delivery. The most prevalent organism in the vaginal environment together with many other facultative and obligate aerobes and anaerobes is lactobacillus. The human genital tract has lower enzymatic which results in less degradation of protein and peptide drugs in the vagina than the gastrointestinal tract.

\section{Vaginal pH}

The vaginal $\mathrm{pH}$ of healthy women of reproductive age is acidic (pH 5.4-5); which is maintained by lactobacillus convert glycogen from epithelial cells to lactic acid. The changes in $\mathrm{pH}$ occur by factors such as age, stages of menstrual cycle, infections and sexual arousal. Menstrual, cervical and uterine secretions and semen act as alkalizing agents and increase the $\mathrm{pH}^{18}$. The vaginal $\mathrm{pH}$ should be controlled for successful vaginal delivery of drugs.

\section{Vaginal absorption of drugs}

Drugs are transported across the vaginal membrane by the trans cellular route, intracellular route or vesicular and receptor mediated transport mechanisms. A physical model of the vaginal membrane as a transport barrier has been described. The physiological factors (e.g. cyclic changes in the thickness and porosity of the epithelium, volume, viscosity and $\mathrm{pH}$ of the vaginal fluid) and physicochemical properties of drugs (e.g. molecular weight, lipophilicity and ionization) affect absorption across the vaginal epithelium. The absorption of drugs, targeted for local action in the vagina, is not desirable ${ }^{20}$.

\section{Vagina as an application site for drug delivery}

The anatomical position, the rich blood supply and the large surface area of the vagina predestines it as an application site for systemic drug delivery. In numerous studies a good permeability to a wide range of compounds including peptides and proteins has been shown. The vaginal route offers a favourable alternative to the parenteral route for some drugs such as bromocriptine, propranolol, oxytocincalcitonin, LHRH agonists, human growth hormone and steroids used in hormone replacement therapy or for contraception the vagina might serve as a better route for the delivery of hormonal contraceptives owing to the lack of drug interactions observed in the gastrointestinal tract. Since now the vagina has been studied 
as a favorable site for the local delivery of drugs, specifically for female-related conditions. Traditionally, the vaginal cavity has been used for the delivery of locally acting drugs such as antibacterial, antifungal, antiprotozoal, antiviral, anti-inflammatory, and spermicidal agents, prostaglandins and steroids. In the past decade, major advancements have been reported in the field of microbicides that are compounds or formulations that can prevent the transmission of sexually transmitted diseases including AIDS. Several microbicides are in different stages of development. The vaginal route also has potential for the uterine targeting of active agents such as progesterone and danazol. The plasma concentrations of vaginally administered progesterone were found to be higher in the uterine artery than in the radial artery, indicating a preferential distribution of progesterone to the uterus. This confirmed the existence of direct local transport from the vagina to the uterus, termed the first uterine pass effect.

\section{Classification of Intra-Vaginal Drug Delivery System \\ - Vaginal rings \\ - Vaginal Tablet \\ - Vaginal Powder \\ - Vaginal Capsule \\ - Vaginal Ointment \\ - Vaginal gel and creams \\ - Vaginal Suppositories}

\begin{abstract}
Vaginal Rings
Vaginal rings are circular ring type drug delivery devices designed to release the drug in a controlled fashion after insertion into the vagina. Advantages of vaginal rings are that it is user controlled, does not interfere with cation, does not require a daily intake of pills and allows continuous delivery of low dose steroids. They are approximately $5.5 \mathrm{~cm}$ diameter with a circular cross section diameter of 4-9 $\mathrm{mm}$ and the ring is inserted in the vagina. In simple vaginal rings, drug is homogeneously dispersed within a polymeric ring $^{6}$. Drug at the surface of the ring is released faster than drug in the inner layer of the ring. Sometimes, drugs in the outermost layer provide an initial burst release. To obtain a constant release of drug from vaginal ring, sandwich or reservoir type rings has been developed. Sandwich type devices consist of a narrow drug containing layer located below the surface of the ring and positioned between a non-medicated central core and a no medicated outer band. In reservoir type rings, drugs are dispersed in a centralized core, which is then encapsulated by a drug free
\end{abstract}

layer of polymer. In a single ring, it is possible to have several cores of different drugs and thereby allowing administration of several drugs from the same device ${ }^{7}$. The rate of drug release can be modified by changing the core diameter or thickness of the no medicated coating. The material for making vaginal ring is usually polymeric in nature. Much of the vaginal ring literature relates to commonly used polymer, poly (dimethylsiloxane) or silicone devices, although other elastomeric polymers such a ethylene vinyl acetate and styrene butadiene block copolymer have been tested in recent years Ethylene vinyl acetate polymers are classified by the content of vinyl acetate. The addition of vinyl acetate units in the polyethylene provides the following advantages: increased flexibility, improved optical properties, greater adhesion, and Further, the clinical acceptability of rings made of ethylene vinyl acetate is very high In evaluating the tolerability of ethylene vinyl acetate no medicated vaginal ring of diameter $54 \mathrm{~mm}$, the acceptability percent among the subjects involved in the study was $91 \%$. The ring was to remain inserted for 21 consecutive days after insertion, permitting temporary removal during coition. Most of the women judged the ring easier to insert and remove. No adverse effects were experienced among the test group during the study period. Vaginal rings are used for contraceptive and hormone replacement therapy. For most contraceptive applications, the rings are placed in the vagina for 21 days followed by a week of ring free period. Nuva Ring is the only combined contraceptive vaginal ring available in the US market. Nuva Ring is a flexible, transparent, contraceptive vaginal ring containing two active components, etonogestrel and ethinylestradiol. The ring releases $120 \mathrm{mg} /$ day of etonogestrel and $15 \mathrm{mg} /$ day of ethinylestradiol over a 3-week period of use. Clinical trials show that Nuva Ring is an effective contraceptive ring with good cycle control and user acceptability FemringR and Estring $R$ are estrogen releasing rings used for estrogen therapy. Femring $R$, which is made up of silicone elastomer, contains acetate derived of estradiol, which is placed in the vagina once every trimester. Estradiol acetate is hydrolyzed to estradiol after being released from the delivery device. Estring $R$ is made of silicone polymers and when inserted in the vagina releases $7.5 \mathrm{mg}$ of estradiol per day (Fig. 2: Vaginal Ring).

\section{Vaginal Tablet}

The manufacturing process of vaginal bioadhesive controlled release matrix tablets 
consist of the preparation of a matrix mixture comprising the pharmaceutically acceptable excipients. The release mechanism is based on drug diffusion through the swollen polymers and progressive erosion /dissolution of the gel matrix. The controlled-release properties of the vaginal tablets may be modified by the presence in the dosage form of soluble and insoluble fillers and by their weight ratio. The insoluble excipients can be selected from the group of microcrystalline cellulose, calcium phosphate tribasic, dibasic calcium phosphate, calcium sulphate and dicalcium phosphate. Either anhydrous or hydrated dicalcium phosphate is preferred. The soluble excipients can be selected from the group of lactose, sorbitol, xylitol, mannitol, amylose, dextrose, fumaric acid, citric acid, tartaric acid, lactic acid, malic acid, ascorbic acid, succinic acid, polyethylene glycols of various molecular weight, soluble hydroxyalkyl celluloses, polyvinylpyrrolidones, gelatins, sodium carbonate and sodium bicarbonate. Mucoadhesive polymers such as polycarbophil, cellulose ethers, chitosan and polyvinylpyrrolidine are used for the preparation of tablet formulations (Fig. 3: Vaginal Tablet).

\section{Vaginal Powder}

Vaginal powder is prepared by dissolving hydroxypropyl cellulose in water with heat. The mixture is slightly cooled and the bisphosphonate is added. The mixture is lyophilized (Fig. 4: Vaginal Powder).

\section{Vaginal Capsule}

Vaginal capsule is prepared by filling the prepared powder into capsules. While the invention has been described in terms of various preferred embodiments, the skilled artisan will appreciate that various modifications, substitutions, omissions and additions may be made without departing the spirit thereof. Accordingly, it is intended that the scope of the present invention be limited solely by the scope of the following claims (Fig. 5: Vaginal Capsule).

\section{Vaginal Ointment}

Vaginal ointment according to the invention comprises an oil and an aqueous phase. For preparation of the ointment the drug selected from the group of compounds consisting of alendronate, clodronate, tiludronate, pamidronate, etidronate, ibandronate, neridronate, residronate, zoledronate or olpadronate is dissolved in the aqueous phase and the oil phase added. Both phases are properly mixed (Fig. 6: Vaginal Ointment).

\section{Vaginal Creams and Gels}

Creams and gels are used for topical delivery of contraceptives and anti-bacterial drugs. These vaginal dosage forms are messy to apply, uncomfortable and sometimes embarrassing when they leak into the undergarments. Further, creams and gels may not provide an exact dose because of non uniform distribution and leakage. The desirable properties of vaginally administered cream or gel against microbicides are acceptability and feasibility. They must be easy to use, non-toxic and non irritating to the mucus membrane. In the treatment of bacterial vaginosis, metronidazole and clindamycin vaginal cream are found to be nearly as effective as orally administered drugs. To evaluate the efficacy of an antibacterial vaginal cream in the treatment of bacterial vaginosis, Lamont et al.Carried out a randomized, placebo controlled 3-day course study during the second trimester of pregnant women. They found that the clindamycin vaginal cream was well tolerated and more efficacious than placebo in the treatment. In the absence of an effective prophylactic antiHIV vaccine or therapy, current efforts are aimed at developing topical intravaginal formulations of anti-HIV agents or microbicides to reduce the mucosal and perinatal virus transmission. Vaginal creamsand gels could be based on the principle of emulsion or hydrogel based drug delivery.

During the past few years, considerable work has been done on the development of hydrogel controlled release drug delivery systems. These hydrogels, when placed in an aqueous environment, swell and retain large volumes of water in their swollen structure and release drug in a controlled fashion. A swelling controlled release hydrogel delivery system for intravaginal administration of an antifungal drug, miconazole, has been reported Hydrogels are hydrophilic polymers that have been cross-linked by means of covalent bonds. A $3 \%$ alginate gel of nonoxynol-9 has been investigated for intravaginal spermicide delivery. In the study, it was shown that spermicidal activity and diffusion of the agent changes with the $\mathrm{pH}$ and osmolarity of the formulation. Recently, gel-microemulsions have been proposed as a nontoxic vaginal formulation. A gel microemulsion based formulation of a spermicide with anti- HIV effect, a vinyl phosphate derivative of zidovudine, has been developed .Multiple intravaginal application of this drug as microemulsion gel formulation did not cause any damage in the vaginal epithelium in a rabbit model. The vaginal gel has also been used for intravaginal vaccine delivery. Intravaginal delivery of cholera vaccine 
showed a greater mucous response in the female genital tract compared to oral administration of the vaccine Antibacterial agents and drugs for cervical ripening and induction of labor are also available as a vaginal gel form. Oxytocin, dinoprostone and misoprostol are commonly used drugs for cervical ripening and induction of labor. Recently9 studied the efficacy of dinoprostone (prostaglandin E2) vaginal gel versus vaginal tablet in the induction of labor. Their retrospective analysis was performed to compare the labor outcomes between women who received dinoprostone vaginal gel (1-2 mg) over a 3- month period and women who were receiving a dinoprostone vaginal tablet (3 $\mathrm{mg}$ ) over the following 3 months. The authors observed no statistically significant differences in labor outcomes between dinoprostone vaginal gel and tablet used in the induction of labor. However, in their analysis, the authors did not compare the safety between the two dosage forms. In another similar study, the efficacy and safety of dinoprostone vaginal insert with vaginal tablet was compared. Women who were requiring labor induction were randomly assigned to receive either a 10 $\mathrm{mg}$ dinoprostone vaginal insert or $3 \mathrm{mg}$ dinoprostone tablet twice at six-hour intervals. The complications for the two dosage forms were tested by the occurrence of uterine hyper stimulation, abnormal fatal heart rate patterns, use of h-2 adrenergic drugs and fatal outcome. The interval from insertion of the induction agent to the onset of regular uterine contractions was similar between the two groups. In seven of eight patients from the group who were receiving the insert and experienced uterine hyper stimulation, removal of the insert was sufficient to stop the hyper stimulation. However, in the group that was receiving tablet, eight out of nine subjects needed medical intervention to end hyper stimulation. An interesting study by10 comparing the efficacy of vaginal misoprostol and dinoprostone vaginal gel for labor induction. The principal outcome measures wereoxytocin requirement in labor, the necessity of analgesia, mode of delivery, time for induction to delivery and neonatal outcome. In misoprostol administered group, a reduced need for oxytocin in labor, but a highly significant reduction in time for induction to delivery was observed compared to the dinoprostone administered group. However, no differences in the requirement of analgesia, mode of delivery, or neonatal outcome were noticed between the two cohorts. In another recent randomized controlled study involving dinoprostone suppository, the vaginal misoprostol administration was found to be
Nevertheless, compared to PGF2-a gel, both misoprostol and dinoprostone suppositories showed a reduced need for oxytocin and shorter labor duration. Since the inception of misoprostol in 1993 for labor induction, the intravaginal administration of this drug hasbeen studied extensively. Recently, there have been several citations in the literature comparing the effectiveness of oral versus vaginal misoprostol delivery. The dose required for the oral delivery of misoprostol is usually 4 times than that of intravaginal dose. However, there have been few conflicting reports too with respect to the efficacy of the route of misoprostol administration. For example, Hall et al. reported that oral misoprostol had the potential to induce labor as safely and effectively as that produced by vaginal misoprostol, whereas a study by11 found that vaginal administration of the drug was more efficacious than the oral route although the oral $(100 \mathrm{mg})$ and vaginal dose (25 mg) as -p;./ XDG HJKJ8well as the intervals of drug administration were the same in both these studies, the results were not similar. This disparity in their observation could be attributed to their principal outcome criterion, which was assessed in each of these studies. In the former study, the key outcome measurement was the time for the start of induction tovaginal delivery, while in the latter study the chief out measurement was the number of women who went on to deliver vaginally within $24 \mathrm{~h}$ of initiation of the first dose of misoprostol. In a specific study evaluating the safety and efficacy of oral versus vaginal misoprostol administration, the investigators found that, although oral misoprostol had similar effects as the vaginal form, the oral administration was associated with higher frequency of high uterine contractility and intervention. In an interesting report, concerning the sublingual use of misoprostol in first-trimester surgical abortion, the authors found that sublingual delivery of misoprostol was an effective alternative to vaginal administration for cervical priming. Although a greater incidence of side effects was observed, the patient acceptability was quite high. From an analysis of different studies performed employing the oral and vaginal routes of misoprostol administration, it appears that the current recommended vaginal misoprostol dose (25 Ag) is efficacious and safer than the $100 \mathrm{Ag}$ oral doses. Also, as rightly noticed by12 different methods of misoprostol administration may not be equivalent with regard to efficacy and safety (Fig. 7: Vaginal Gel and Fig. 8: Vaginal Cream). 


\section{Suppositories}

A large number of vaginal medications are available in the form of tablets or suppositories. Some authors use the terms pessaries and suppositories interchangeably and consider vaginal tablets as a separate dosage form. These vaginal formulations are designed to melt in the vaginal cavity and release the drug for several hours. Suppository systems are now most commonly used to administer drugs for cervical ripening prior to childbirth and local delivery of drugs. Drugs that are administered as suppository include dehydroepiandrosteronesulfate for ripening effect on the uterine cervix, Miconazole for vaginal candiasis and progesterone for hormone replacement therapy. Vaginal tablets may contain binders, disintegrate and other excipients that are used to prepare conventional oral tablets. It has the advantage of easy of manufacture and insertion. Mucoadhesive polymers are sometimes used in vaginal tablet formulation to increase vaginal residence time. Drugs that are administered as vaginal tablets include itraconazole, clotrimazole and prostaglandins. Presence of hydrophobic and release retarding materials may decrease the absorption of a drug from a vaginal formulation. Too hydrophobic drugs may not be suitable for vaginal tablets. Presence of penetration enhancers such as surfactants, bile salts can significantly enhance absorption (Fig. 9: Vaginal Suppositories).

\section{Vaginal Films}

Another mucoadhesive solid dosage form is film $^{33}$. Vaginal films are polymeric drug delivery systems shaped as thin sheets, usually ranging from 220 to 240 micrometre in thickness. These systems are often square (approximately $5 \mathrm{~cm} \times 5 \mathrm{~cm}$ ), colourless and soft, presenting a homogenous surface. Vaginal films are produced with polymers such as polyacrylates, polyethylene glycol, polyvinyl alcohol and cellulose derivatives. A bioadhesive hot-melt extruded film is used for topical and mucosal adhesion applications. The film is made from a precursor composition containing a water-soluble or water-swellable thermoplastic polymer, preferably HPC and/or PEO and a bioadhesive polymer. The film can also contain a therapeutic agent, preservative, buffering agent, antioxidant, super-disintegrate or absorbent, flavorant, colorant, waterinsoluble polymer, organic acid, surfactant, film modifier and/or cross-linking agent. A mucoadhesive film formulation which is suitable for delivery of therapeutic agents to vaginal mucosa has been developed ${ }^{34}$. Film composition for delivery of pharmacologically effective agents topically to vaginal mucosa comprises polymer which is hydrophilic, hydrophobic or mixture of both. The polymer is selected from the group consisting of hydroxypropyl methylcellulose, gelatin, alginic acid, alginic acid sodium salt, pectin, collagen, poloxamer, carbopol, microcrystalline cellulose, polyacrylic acid, polyethylene glycol and polypropylene glycol. The film has a controllable rate of gelling, swelling and degradation and is preformed into a device or is applied as a coating to the surface of a more complex drug delivery system. $\mathrm{pH}$-Responsive film for intravaginal delivery of a beneficial agent has been prepared for the intravaginal administration of prophylactic and therapeutic agents $^{35}$ (Fig. 10: Vaginal Suppositories).

\section{Mucoadhesive Drug Delivery Systems}

Mucoadhesion is a topic of interest in the design of drug delivery systems. Various types of formulations have been widely used to prolong the residence time of the dosage form at the site of application. They were introduced in 1947 and recent reports have suggested that the market share of mucoadhesive drug delivery systems has been increasing. Mucoadhesive formulations play a key role in the release of the drug through their attachment to the vaginal mucosa and are currently being utilized for controlled release. Mucoadhesion can be defined as a state in which two components, of which one is of biological origin are held together for extended periods of time by the help of interfacial forces. Mucoadhesion is used when the bond is formed with a mucosal surface. Mucoadhesive drug delivery systems includes the following,

- Buccal delivery system

- Oral delivery system

- Vaginal delivery system

- Rectal delivery system

- Nasal delivery system

- Ocular delivery system

\section{Mechanism of Mucoadhesion}

As stated, mucoadhesion is the attachment of the drug along with a suitable carrier to the mucous membrane. Mucoadhesion is a complex phenomenon which involves wetting, adsorption and interpenetration of polymer chains. Mucoadhesion has the following mechanism

1. Intimate contact between a Mucoadhesive and a membrane( wetting or swelling phenomenon)

2. Penetration of the mucoadhesive into the tissue or into the surface of the mucous membrane(interpenetration).

\section{Characteristics of Mucoadhesive Polymer}

1. Cationic and anionic polymers bind more effectively than neutral polymers. 
2. Poly-anions are better than polycations in terms of binding/ potential toxicity, and further, that water-insoluble polymers give greater flexibility in dosage form design compared with rapidly or slowly dissolving water-soluble polymers.

3. Anionic polymers with sulfate groups bind more effectively than those with carboxylic groups.

4. Degree of binding is proportional to the charge density on the polymer.

5. Highly binding polymers include carboxyl methyl cellulose, gelatin, hyaluronic acid, carbopol and polycarbophyl.

\section{Classification of dosage Form of Mucoadhesive \\ 1) Mucoadhesive Gel Formulations}

Mucoadhesive semi-solid formulations are able to facilitate intimate contact with the underlying absorption surface and improve the bioavailability of drugs. Rheological properties of gels are important for their retention on the vaginal surface, which are fundamental to their efficacy. The remarkable elastic character and the improvement of the rheological properties of the mucoadhesive gel prolong the residence time at the application site. For these kinds of formulations, selection of correct viscosity of the formulation is important in order to provide adequate retention and distribution in the vagina.

\section{2) Mucoadhesive Tablet Formulations}

Vaginal mucoadhesive tablet formulations are important alternatives to conventional vaginal formulations and are particularly useful for the therapy of insistent vaginal infections as they reduce the required dose frequency, provide easy application and therefore increase patient compliance. Chitosan is used in mucoadhesive tablets for vaginal delivery of metronidazole. The polymer matrix containing a mixture of chitosan, Carrageenan, xanthun gum, polyox WSR 303, Hydroxypropylmethyl cellulose, magnesium staterate, microcrystalline cellulose provides an adequate release of metronidazole for $8 \mathrm{~h}$ and maximum adhesion was also obtained with minimum pressure applied.

\section{3) Mucoadhesive Suppositories}

Among the solid vaginal preparations, suppositories have advantages as they facilitate the application due to their slippery and smooth surface. To provide a long term therapeutic concentration of miconazole following a single dose, hydrogel based suppositories were prepared with polyvinyl alcohol and the effect of the time of the freezing and thawing cycles was investigated vis-a-vis the swelling property of the hydrogels outcome. It was observed that an increase in the number of freeze-thaw cycles reduced the equilibrium swelling of polyvinyl alcohol hydrogel. This reduction in equilibrium swelling was due to an increase in the degree of crystallinity of the hydrogel. The release of the drug was continued beyond three days and the Fickian diffusion mechanism of release was found predominant.

\section{4) Mucoadhesive Particulate Formulations}

Although several studies have focused on mucoadhesive drug delivery systems in the form of tablets, films, patches, and gels for mucosal routes, very few reports on mucoadhesive microparticles are available. Hyaluronan esters (HYAFF) have opened new avenues for mucoadhesive vaginal formulations. Due to their biocompatibility and controllable degradation rate HYAFF microspheres have been used for localized drug delivery of steroids, analgesics, antiinflammatory and anti-infectives. Recent studies have indicated that a number of polymeric delivery systems such as mucoadhesive microspheres possess significant potential for the development of vaginally administered vaccines.

\section{5) Mucoadhesive Film Formulations}

Thin films have been found to be suitable physical forms for vaginal drugs and peptide delivery. To develop an efficient female controlled drug delivery system against sexually transmitted diseases, polymeric films containing sodium dodecyl sulfate were prepared with various compositions of Carbopol 934P, hydroxypropylmethylcellulose and polyethyleneglycol. It was demonstrated that the films made of Carbopol, hydroxypropylmethylcellulose and polyethyleneglycol were colorless, thin and soft, also had sufficient strength to withstand mechanical damage during production, handling and application for a female controlled drug delivery system. An increase in Carbopol content elevated the tensile strength and swelling ratio of the films but decreased the contact angle, erosion rate and sodium dodecyl sulfate release rate from the films. The films containing $0.25 \% \quad(\mathrm{w} / \mathrm{v})$ polyethyleneglycol as well as $0.75 \%(\mathrm{w} / \mathrm{v})$ of Carbopol/ Hydroxypropyl methylcellulose remained on the vaginal tissue for up to 6 hours.

\section{Table 1: Marketed mucoadhesive vaginal Formulation}




\begin{tabular}{|c|c|c|}
\hline Drug (Product) & Dosage form & Indication \\
\hline Progesterone (Prochieve®) & Gel & Infertility, secondary amenorrhoea \\
\hline Nonoxynol-9 (Gynol II@) & Gel & Contraception \\
\hline Acid-buffering (Buffer Gel®) & Gel & Contraception \\
\hline Dinoprostone (Prostin E2ß) & Gel & Induction of labour \\
\hline 2-Naphthalene sulfonic acid (PRO 2000/5®) & Gel & Contraception \\
\hline Chlorhexidine (Clomirex®) & Gel & Bacterial infection \\
\hline Estradiol (Vagifem®) & Tablet & Atrophic vaginitis \\
\hline Nonoxynol-9 (Conceptrol囚) & Gel & Contraception \\
\hline Acid-buffering (Aci-Jel@) & Gel & $\begin{array}{c}\text { Restoration and maintenance of } \\
\text { vaginal acidity }\end{array}$ \\
\hline Nonoxynol-9 (Advantage S®) & Gel & Contraception \\
\hline Metronidazole (Hycore -V®) & Hydrogel pessary & Anti-fungal agent \\
\hline SPL7013 gel (Vivagel囚) & Dendrimer & $\begin{array}{c}\text { Treatment of sexually transmitted } \\
\text { diseases }\end{array}$ \\
\hline Clotrimazole (Mycelex-G®) & Bioadhesive tablet & Vaginal candidiasis \\
\hline Acid-buffering (RepHresh®) & Gel & Bacterial vaginosis \\
\hline
\end{tabular}

\section{In vitro and In vivo Studies}

These studies include the determination of drug release and mucoadhesive characteristics in addition to various physical and chemical properties of formulations ${ }^{36}$. The release characteristics of a drug from a vaginal formulation can be determined in simulated vaginal fluid $(\mathrm{pH} \mathrm{4.2)}$ and in various dissolution media ( $\mathrm{pH}$ range 2-12) by different types of diffusion cells with certain modifications and a vaginal dissolution tester ${ }^{37}$. The mucoadhesive strength of the vaginal formulation can be measured by various techniques ${ }^{38}$. In vivo studies are conducted in different animal models to assess efficacy, distribution, spreading and retention of formulations in the vagina. Gamma scintigraphy and colposcopy ${ }^{39}$ are desirable techniques for assessing the distribution, spreading and retention of vaginal formulations in sheep and humans. However, the significance of these findings is debatable. Two imaging techniques are being developed to measure the degree of coverage in the vaginal vault: magnetic resonance imaging (MRI) and an intravaginal optic probe ${ }^{40}$. Several animal models such as sheep, rats, rabbits, rhesus monkeys, macaque monkeys, dogs and mice have been used in different studies in the development of vaginal formulations ${ }^{41}$. White rabbits are used for primary irritation and sub chronic toxicity testing. Recently developed vaginalectocervical (VEC) tissue models will serve as useful, highly reproducible, non-animal tools to assess the irritation due to vaginal care product $^{42}$.

\section{CONCLUSION}

The vaginal route act as a potential route for the delivery of therapeutically important molecules, such as microbicides and novel Mucoadhesive vaginal drug delivery systems includes vaginal tablets, vaginal films, vaginal creams, vaginal gels, Suppositories or Pessaries. These novel systems would enhance the delivery of many drugs offering better therapeutic outcomes. The vaginal route has been used for the local application of drugs, but is now becoming a potential route for noninvasive, controlled transmucosal delivery of both local and systemic therapeutically active compounds. The safety and efficacy of vaginal administration have been well established. Novel mucoadhesive vaginal delivery systems overcome some of the key limitations associated with conventional delivery of vaginal drugs. From this review it was evident that the vaginal drug delivery is a promising area for continued research on the delivery of microbicides that can prevent HIV infection and other transmission of sexually transmitted diseases. 


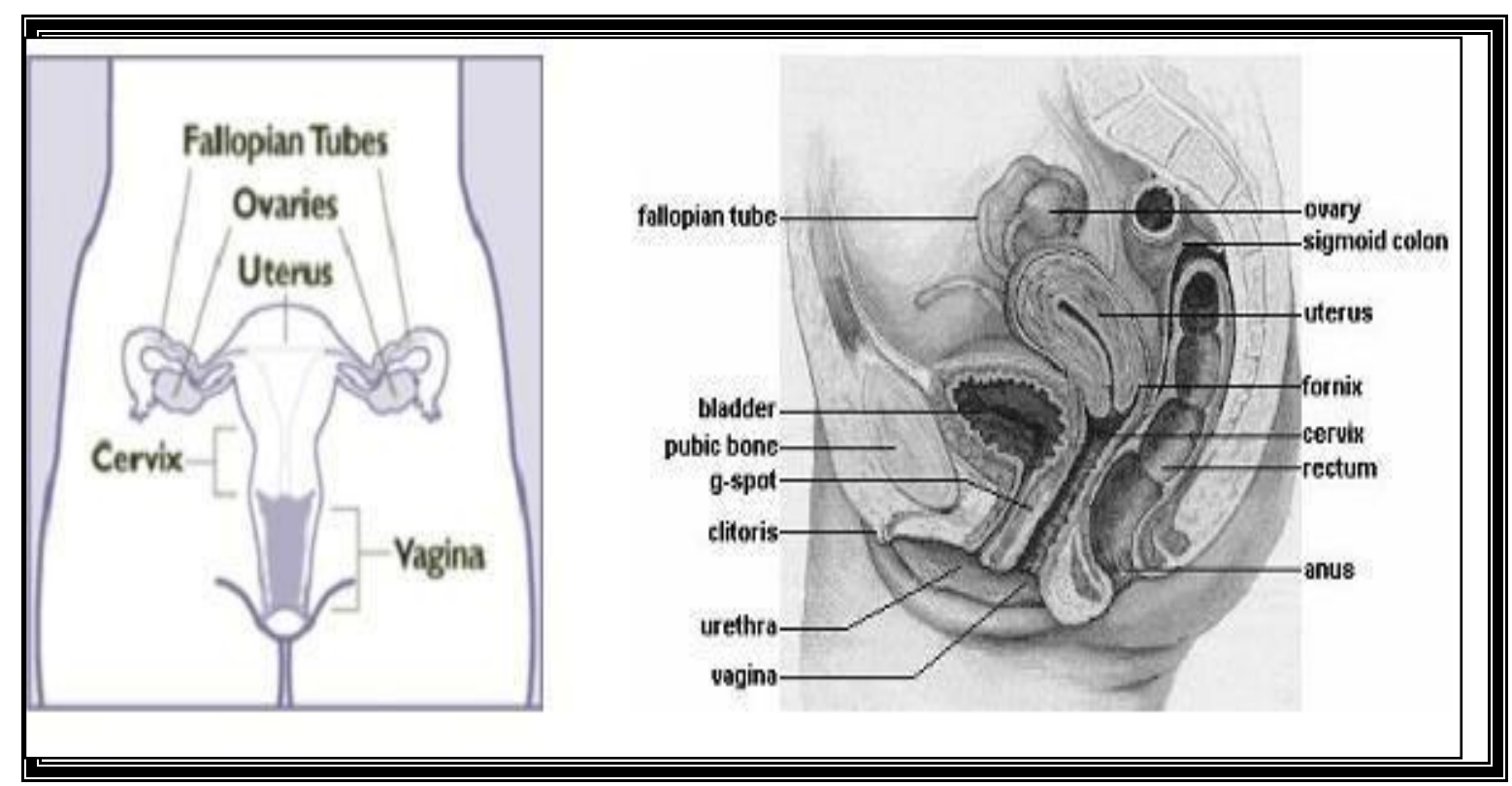

Fig. 1: Schematic drawing of the vagina

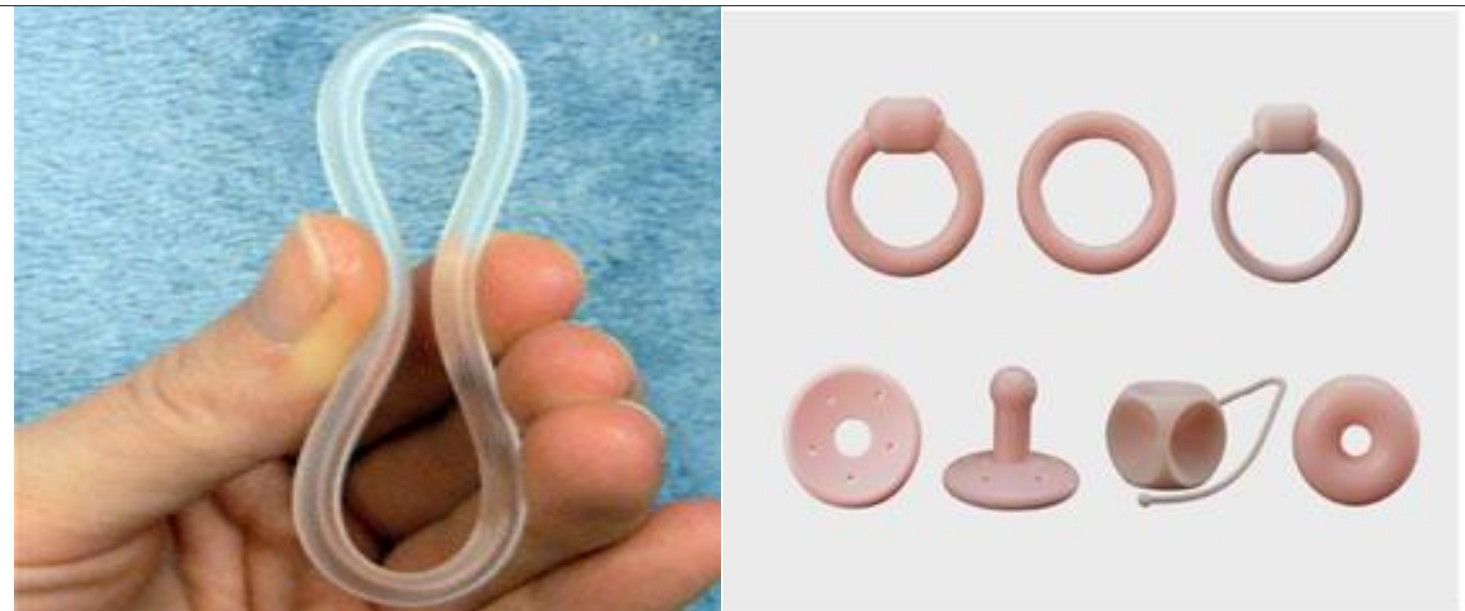

Fig. 2: Vaginal Ring

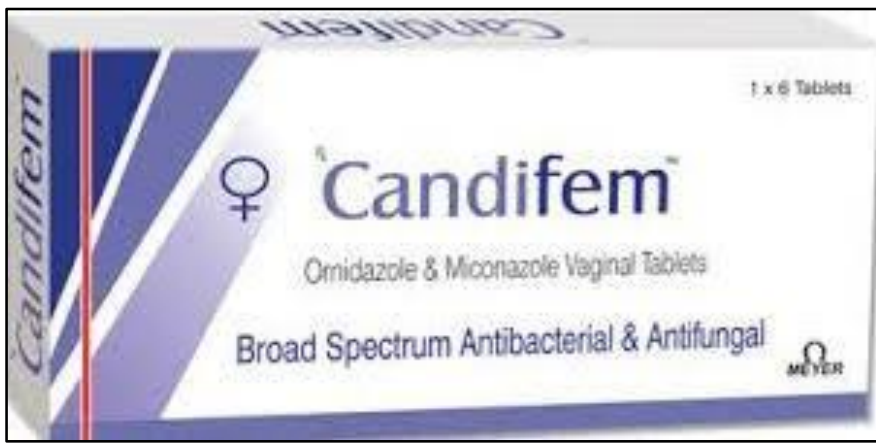

Fig. 3: Vaginal Tablet 


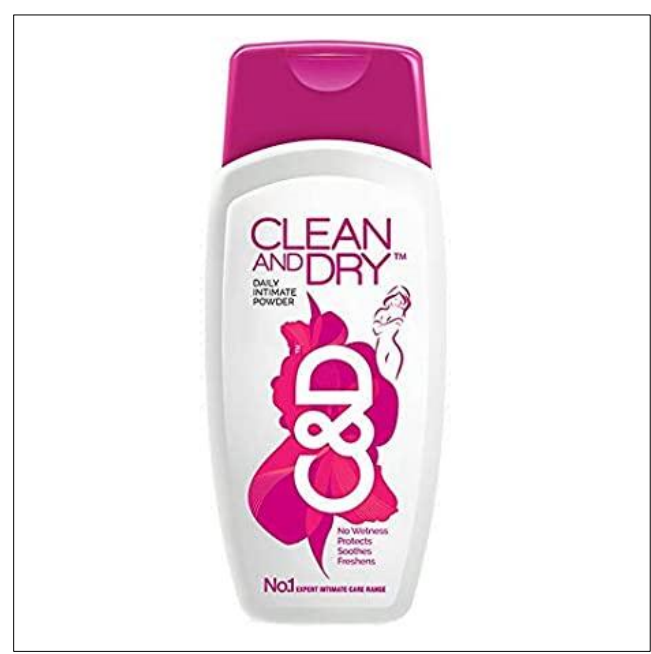

Fig. 4: Vaginal Powder

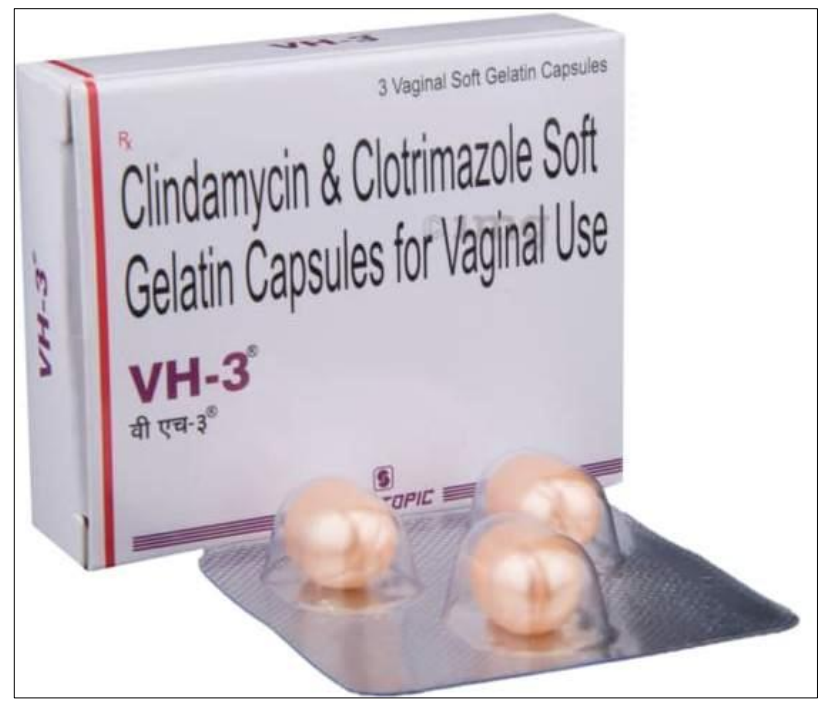

Fig. 5: Vaginal Capsule

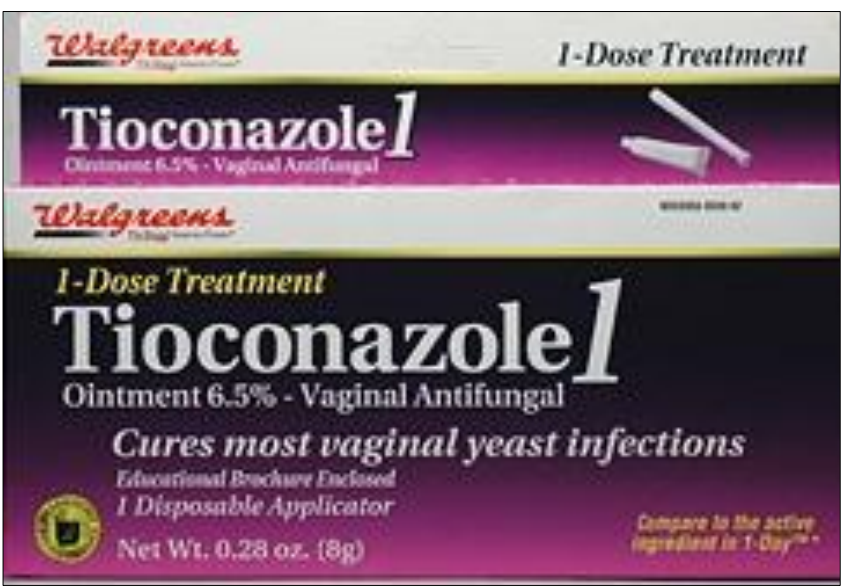

Fig. 6: Vaginal Ointment 


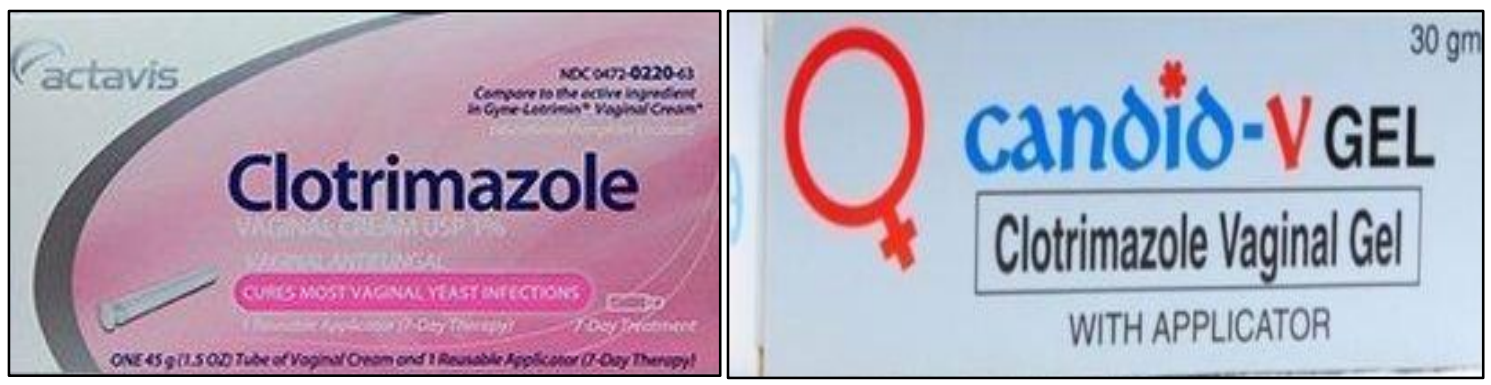

Fig. 7: Vaginal Gel

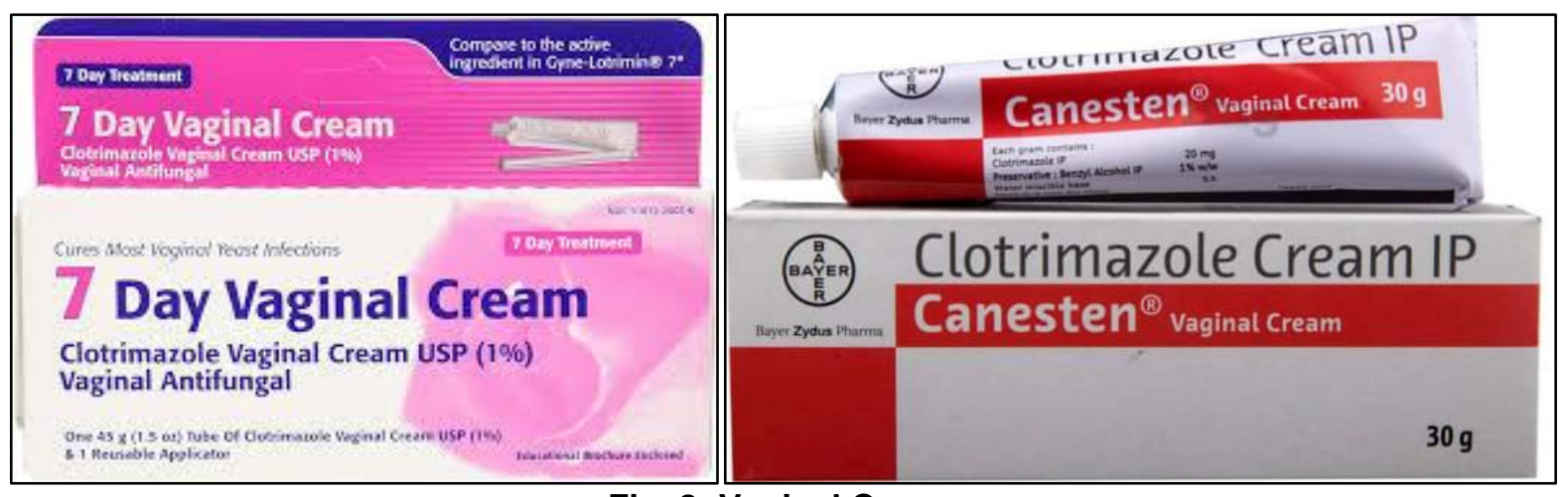

Fig. 8: Vaginal Cream

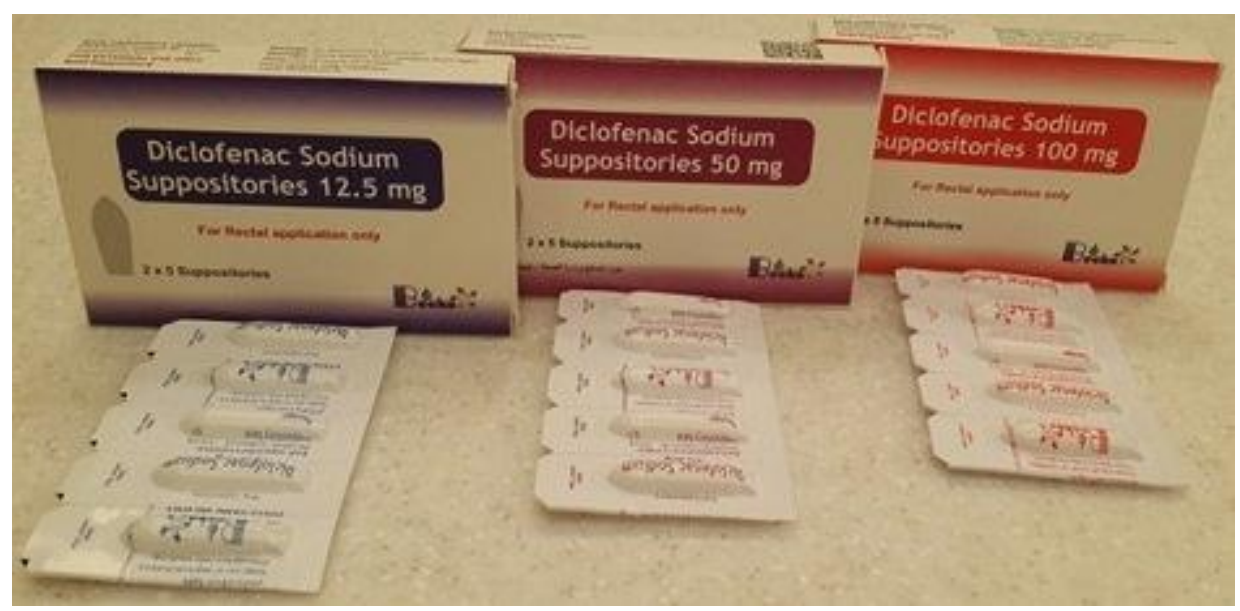

Fig. 9: Vaginal Suppositories 


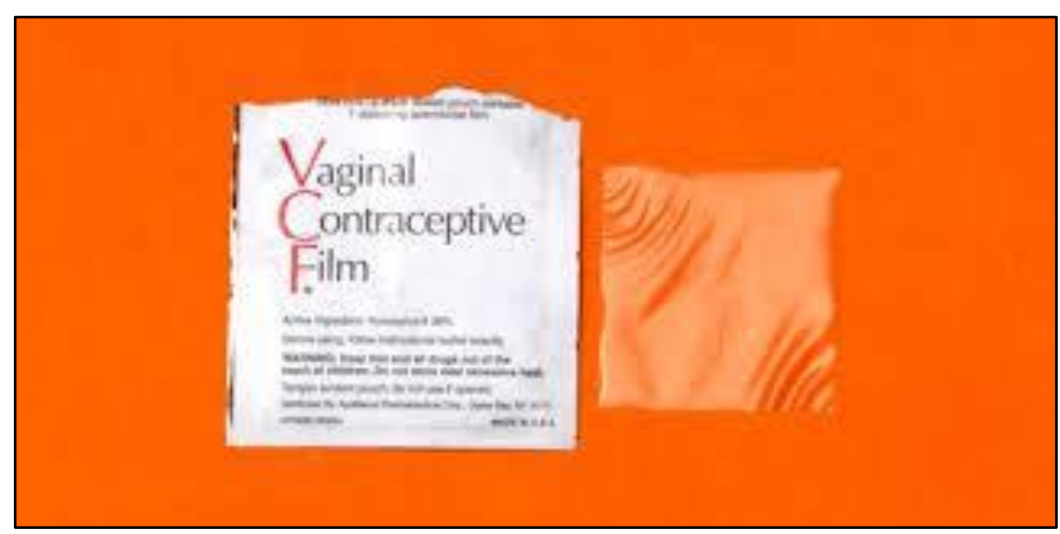

Fig. 10: Vaginal Suppositories

\section{REFERENCES}

1. Alexander $\mathrm{NJ}$, Baker E, Kaptein M and Miller L. Why consider vaginal drug administration, Fertility and Sterility. 2004;82:1-12.

2. Woolfson AD, Melcolm RK and Gallagher R. Drug delivery by the intravaginal route. Crit Rev Ther Drug Carr Syst. 2000;17:509-55.

3. Washington $\mathrm{N}$, Washington $\mathrm{C}$ and Wilson CG. Vaginal and intrauterine drug delivery in Physical pharmaceutics: barriers to drug absorption, (N. Washington, C. Washington \& CG. Wilson, eds.) Taylor and Francis, Landon. 2001;271-81.

4. Richter $\mathrm{K}$ and Frick $\mathrm{H}$. Anatomy of visceral fascia of the pelvis from the didactical view point (In German), Geburtshilfefrauenheilkd. 1985; 45:282-87.

5. Soper DE. Genitourinary infections and sextually transmitted disease, in "Novak's gynecology", (S. Berek, EY.Adashi, PA. Hillard, eds.) Willams\& Wilkins. 2007;132-57.

6. Nova'k A, Loge C De La, Ebetz $L$ and Van Der Meulen EA. The Combined Contraceptive Vaginal Ring, NuvaRing: An International Study of User Acceptability, Contraception. 2003;67:187-194.

7. Harwood B and Mishell DR. Contraceptive Vaginal Ring. Semin Reprod Med. 2001;19:381-390.

8. Shetty A, Livingston I, Acharya $S$ and Templeton A. Vaginal Prostaglandin E2 gel Versus Tablet in the Induction of Labour at Term. A Retrospective Analysis. J Obstet Gynaecol. 2004;24:243-246.

9. Semmens JP, Tsai CC, Semmens EC and Loadholt CB. Effects of estrogen therapy on vaginal physiology during menopause, Obstet Gynecol. 1985;66:8-15.

10. Danielian P, Porter B, Ferri N, Summers $J$ and Templeton $A$. Misoprostol for induction of Labour at Term: A More Effective Agent Than Dinoprostone Vaginal Gel Br. J Obstet Gynaecol. 1999;1106:793- 797.

11. Shetty A, Livingstone I, Acharya S, Rice P, Danielian P and Templeton A. Oral Misoprostol (100 Ag) Versus Vaginal Misoprostol (25 Ag) In Term Labor Induction: A Randomized Comparison. Acta Obstet. Gynecol. Second. 2003;82:1103-1106.

12. I'nal MM, K. Ertopc UA and Arici I and Zelmas O. The Effect of Oral Versus Vaginal Misoprostol onCervical Dilatation in first TrimesterAbortion. A Double-Blind,Randomized Study. Eur $J$ Contract. Reprod. Health Care. 2003;8:197-202.

13. Bhowmik D, Chiranjib Biswajit, Dubey V, Tripathi KK and Kumar Sampath KP. Recent advances in Intrauterine Drug Delivery Systems. Scholars Research Library. 2010;1(1):70-75.

14. Ashok V, Kumar RM, Murali $D$ and Chatterjee A. A Review on Vaginal Route as a systemic drug Delivery. Critical Review in Pharmaceutical sciences. 2012;1(1):1-19.

15. Dobaria N, Mashrav R and Vadia NH. Vaginal Drug Delivery System. A Review of Current Status. East and Central African Journal of pharmaceutical Sciences. 2007;10:313.

16. Jain NK. Controlled and Novel Drug Delivery. CBS Publishers and Distributors, Daryaganj, New Delhi, 1st Ed. 1997;353-377.

17. Punitha $E$, Anbazhagan S, Sathya B and Christopher Vimalson D. Recent approaches in vaginal drug delivery 
systems, International Journal of Research in Pharmacy and Pharmaceutical 2018;3(2):97-106.

18. Thrakaramarao $\mathrm{CH}$, Vijyalakshmi NG and Akila S. Application of Vaginal drug delivery. A review; International Journal of comprehensive pharmacy. 2013;4(1):1-4.

19. Krishna SV, Ashok $V$ and Chatterjee A. A Review On Vaginal Drug Delivery Systems, International Journal of Biology, Pharmacy And Allied Sciences, IJBPAS. March. 2012;1(2): 152-167.

20. Hwang S, Wada EO, Yotsuanagi T, Suhardja I. NFH HO, Flynn GL and Higuchi WI. Systems approach to vaginal delivery of drugs: II. In situ vaginal absorption of unbranched aliphatic alcohols. J Pharm Sci. 1977;65:1574-1578.

21. Rahamatullah Shaikh, Thakur Raghu Raj Singh and Martin James Garland. A David Woolfson, and Ryan F. Donnelly, Mucoadhesive drug delivery systems. Journal of Pharmacy and Bioallied Sciences. 2011;3(1):89100.

22. Schmidt EH and Beller FK. Biochemistry of the vagina, in: ESE Hafez TN. Evans (Eds.), Human reproductive medicine: the human vagina, North-Holland Publishing, New York. 1978;2:139-149.

23. Garg S, Tambwekar K, Vermani K, Garg A and Zaneveld LJD. microbicides for prevention of HIV infection. Pharm Technol. 2001;25:1424.

24. Kandarapu R, Grover V and Garg S. Evaluation of the compatibility of ketorolac tromethamine with selected polymers and common tablet excipients by thermal and isothermal stress testing. STP Pharm Sci. 2001;11:449-457.

25. Rosemary A, Giselle M, Atice L, Elizabeth A, Edith W and Ingemar S. A comparative study of safety and efficacy of continuous low dose oestradiol released from a vaginal ring compared with conjugated equine oestrogen vaginal cream in the treatment of postmenopausal urogenital atrophy. BJOG. 1996;103(4):351-8.

26. McGregor JA, French JI, Jones W, Milligan K, McKinney $\mathrm{PJ}$ and Patterson E. Bacterial vaginosis is associated with prematurity and vaginal fluid mucinase and sialidase: results of a controlled trial of topical clindamycin cream. Amer J Obst and Gyne. 1994;170(4):1048-60.

27. Bygdeman M and Swahn ML. Replens versus dienoestrol cream in the symptomatic treatment of vaginal atrophy in postmenopausal women. Maturitas. 1996;23(6):259-63.

28. Kicovic PM, Cortes-prieto J, Milojevic, Haspels AA and Aljinovic A. The treatment of postmenopausal vaginal atrophy with ovestin vaginal cream or suppositories. clinical, endocrinological and safety aspects. Maturitas December. 1980;2(4):27582.

29. Dickerson J, Bressler R, Christian CD and Hermann HW. Efficacy of estradiol vaginal cream in postmenopausal women. Clin Pharmaco and Therap. 1979;26(4):502-7.

30. Das Neves J and Bahia MF. Gels as vaginal drug delivery systems. Int $\mathrm{J}$ Pharm. 2006;318:1-14.

31. Hussain A and Ahsan F. The vagina as a route of systemic drug delivery. $\mathrm{J}$ Controlled release. 2005;103:301-13.

32. Vaginal suppositories and pessaries available from (British Pharmacopoeia).

33. Füsun Acartürk. Mucoadhesive Vaginal Drug Delivery Systems. recent Patents on Drug Delivery and Formulation. 2009;3(3):193-205.

34. Pauletti GM, Desai KJ, Roweton SL, Harrison DC and Sanders LM. lontophoretic device and method of delivery of active agents to biological. 2007. interface. WO2007041118A1.

35. Maniar $\mathrm{M}$ and Parandoosh S. $\mathrm{pH}-$ responsive film for intravaginal delivery of a beneficial. 2005. Agent WO2005013906A3.

36. Valenta C, Kast CE and Harich I. Development and in vitro evaluation of a mucoadhesive vaginal delivery system for progesterone. J Control Rel. 2001;77(3):323-332.

37. Alamdar Hussain and Fakhrul Ahsan. The vagina as a route for systemic drug delivery, Journal of Controlled release. 2005;103;301-313.

38. Genc L, Oguzlar C and Guler E. Studies on vaginal bioadhesive tablets of acyclovir. Pharmazie. 2000;55(4):297-299.

39. Patton DL. STD. 1998;25:421-424.

40. Barnhart KT, Pretorius ES and Shera DM. The optimal analysis of MRI data 
to quantify the distribution of a microbicide. Contraception. 2006;73(1):82-87.

41. Castle PE, Whaley KJ and Cone RA. Contraceptive testing of vaginal agents in rabbits. Contraception. 1998;58(1):51-60.
42. Ayehunie S, Cannon $C$ and Lamore S. Organotypic human vaginalectocervical tissue model for irritation studies of spermicides, microbicides, and feminine-care products Toxicol. In Vitro. 2006;20(5):689-698. 